\title{
On Robustness to Noise of Least Squares Based Adaptive Control*
}

\author{
J. B. MOORE† and G. CASALINO +
}

\begin{abstract}
Global convergence results are presented for the self-tuning regulator based on least squares parameter estimation and minimum variance control. These results are also generalized to the adaptive pole assignment case, and focus on robustness to unmodelled colored noise.
\end{abstract}

Key Words Adaptive control; adaptive systems; control system analysis; least-squares estimations.

\begin{abstract}
Current engineering practice for adaptive control schemes is to base the design on globally convergent schemes for simple plant models. An important class of such schemes uses least squares estimation of assumed simple input-output models and constructs the controller using the parameter estimates. This paper studies the robustness of such schemes to the presence of unmodelled plant coloured noise. Such noise is sometimes an adequate model for unmodelled plant dynamics.

The theory of the paper makes a connection between the least squares parameter error equations and those associated with extended least squares using a posteriori noise estimates for which there are known global convergence results. For the case of adaptive minimum variance control of minimum phase plants, this connection permits stronger convergence results than those hitherto derived from the theory of extended least squares based on a priori noise estimates.
\end{abstract}

\section{INTRODUCTION}

THE PURPOSE of this paper is to provide the crucial first stages towards a global convergence theory for recursive least squares (RLS) based adaptive control schemes applied to autogressive moving average exogenous input (ARMAX) models. The main results show connections to known convergence theory for extended least squares (ELS) schemes with a posteriori noise estimates--these giving stronger results than for ELS schemes based on $a$ priori noise estimates.

Consider as a plant the autoregressive moving average exogenous input (ARMAX) scalar variable signal model

$$
A^{p} y_{k}=B^{p} u_{k}+C^{p} w_{k}
$$

\footnotetext{
* Received 18 February 1986; revised 23 July 1986; revised 22 September 1986. The original version of this paper was not presented at any IFAC meeting. This paper was recommended for publication in revised form by Associate Editor Y. Sunahara under the direction of Editor P. C. Parks.

t Department of Systems Enginecring, Research School of Physical Sciences, Australian National University, G.P.O. Box 4. Canberra, A.C.T. 2601, Australia.

$\ddagger$ Department di Informatica, Sistemistica e Telematica, University of Genoa, Genoa, Italy.
}

where $u_{k}$ is the control input sequence, $y_{k}$ is the output and $w_{k}$ is a zero mean, bounded variance "white" noise disturbance. Here $u_{k}, y_{k}$ are measurable and $w_{k}$ is nonmeasurable. Also, with $q^{-1}$ the unit delay operator, $A^{p}=1+a_{1}^{p} q^{-1}+\cdots a_{n}^{p} q^{-n}$, $B^{p}=b_{1}^{p} q^{-1}+\cdots b_{m}^{p} q^{-m}$ and $C^{p}=1+c_{1}^{p} q^{-1}+\cdots$ $\mathrm{cPq}^{-1}$. Without loss of generality, assume $C^{p}$ is minimum phase. In the first instance assume also that $A^{p}, B^{p}$ are coprime.

One class of adaptive controller for models such as (1.1) implements on-line an extended least squares (ELS) recursive estimation of $A^{p}, B^{p}, C^{p}$ and implements a controller with parameters calculated on-line from the parameter estimates $\hat{A}_{k}^{p}, \hat{B}_{k}^{p}, \hat{C}_{k}^{p}$ (Kumar and Moore, 1982; Moore, 1983, 1985). The controller can be designed based on the certainty equivalence principle, or better using central tendency adaptive control (Ryall et al., 1985). In the convergence theory for such schemes, the noise model is restricted as

$$
\left[\left(C^{p}\right)^{-1}-\frac{1}{2}\right] \text { strictly positive real }
$$

which is satisfied if the noise $C^{p} w_{k}$ is "close" to white (Moore, 1982). Simulation studies show that failure of (1.2) typically results in the ELS scheme giving drifting parameter estimates. Techniques have been devised to side-step the above positive real condition by adding dither signals within the calculations (not necessarily within the control loop), and performing on-line spectral factorization (Moore, 1982).

In current practice, engineers tend to avoid ELS based schemes, being more comfortable with recursive least squares (RLS) schemes constructed with deterministic signal models in mind, or stochastic models (1.1) driven by white noise as when $C^{p}=1$. It is known that adaptive control schemes so designed are frequently robust to the presence 
of colored noise $C^{p} w_{k}$. The self-tuning regulator (STR) of Åström and Wittenmark (1973), designed to tune to a minimum variance controller when applied to minimum phase plants, is such a scheme. Existing global convergence theory for the STR applied to ARMAX models (1.1) (Moore and Bitmead, 1984) views the STR as an ELS scheme for the reorganized model

$$
\begin{aligned}
& A y_{k}=B u_{k}+(C-1)\left(w_{k}-y_{k}\right)+w_{k} \\
& A=1+A^{p}-C^{p}, \quad B=B^{p}, \quad C=C^{p} .
\end{aligned}
$$

The ELS scheme has a priori noise estimates $\hat{w}_{k / k-1}$ which satisfy $\hat{w}_{k k-1}=y_{k}$ under the control action

$$
\hat{B}_{k} u_{k}=\left(\hat{A}_{k}-1\right) v_{k}
$$

Here $\hat{A}_{k}, \hat{B}_{k}$ are the recursive least squares (RLS) parameter estimates which are calculated recursively in terms of a regression vector, defining $r=\max (n, l)$ :

$\psi_{k}^{\prime}=\left[y_{k-1} y_{k-2} \ldots y_{k-r} u_{k-1} u_{k-2} \ldots u_{k-m}\right]$.

Denoting $A=1+a_{1} q^{-1}+\cdots a_{n} q^{-r}, B=b_{1} q^{-1}+$ $\cdots b_{m} q^{-m}, \theta^{\prime}=\left[a_{1} a_{2} \ldots a_{r} b_{1} b_{2} \ldots b_{m}\right]$ then estimates $\hat{A}_{k}, \hat{B}_{k}$ are given from

$\hat{\theta}_{k}=\hat{\theta}_{k-1}+P_{k} \psi_{k}\left(y_{k}-\hat{y}_{k / k-1}\right), \quad \hat{y}_{k / k-1}=\psi_{k}^{\prime} \hat{\theta}_{k-1}$ $P_{k}=P_{k-1}-P_{k-1} \psi_{k} \psi_{k}^{\prime} P_{k-1} /\left(1+\psi_{k}^{\prime} P_{k-1} \psi_{k}\right)$

with suitable initial conditions $P_{0}>0$. The ELS convergence results of Moore and Bitmead (1984) assume the noise condition (1.2) and that the variance $\sigma_{w}^{2}$ of $w_{k}$ satisfies $\sigma_{w}^{2}>0$. In essence they tell us that the closed-loop system achieves the characteristic polynomial $q B^{p} C^{p}$ for arbitrary $C^{p}$ satisfying (1.2).

The convergence results for ELS schemes based on a priori noise estimates $\hat{w}_{k \mid k-1}$ are not as strong as those for ELS schemes based on a posteriori noise estimates $\hat{w}_{k \mid k}$. In the latter case, for example, convergence rates can be guaranteed but not so in the former case.

One contribution of this paper which emerges as a by-product of the main theory is to demonstrate a connection of the STR scheme above with an ELS scheme based on a posteriori noise estimates. With such a connection, stronger convergence results can be obtained than in the present theory (Moore and Bitmead, 1984), in that convergence rates can be established.

What then about more general RLS based adaptive control schemes assuming models (1.1) with $C^{p} w_{k}$ white, but applied when $C^{p} w_{k}$ is colored? In this paper a natural generalization of the STR concept of Áström and Wittenmark (1973) is considered. The objective is to achieve closed-loop poles at the zeros of $Q C^{p}$, for some minimum phase polynomial $Q$, invariant of the noise color $C^{p}$ of the model (1.1). The polynomial $Q$ may be specified a priori as in adaptive pole assignment. Alternatively, as in adaptive linear quadratic Gaussian (LQG) schemes, there can be on-line calculations which in effect assign closed-loop poles depending on the performance index and plant parameter estimates. The theory of Casalino et al. (1985) is pertinent to such schemes and is now briefly summarized, and some aspects made more precise.

Consider a feedback control law applied to (1.1), with $R, S$ polynomial finite in $q^{-1}$, as

$$
R u_{k}=S_{y_{k}}, \quad R \text { monic. }
$$

An important subclass is when the closed loop system has an autoregressive exogenous input (ARX) "implicit" representation of the form

$$
A y_{k}=B u_{k}+w_{k}, \quad R u_{k}=S y_{k}
$$

with $A=1+a_{1} q^{-1}+\cdots, \quad B=h_{1} q^{-1}+b_{2} q^{-2}$ $+\cdots$. polynomials in $q^{-1}$ of finite dimension.

Theorem 1.1 (See Casalino et al., 1985). The controller (1.7) applied to the plant (1.1) allows a closedloop ARX “implicit" representation (1.8) iff there exists a polynomial operator in $q^{-1}$, denoted $Q$, such that $Q\left(q^{-1}=0\right)=1$ and

$$
\begin{gathered}
A^{p} R-B^{p} S=Q C^{p}, \\
A R-B S=Q .
\end{gathered}
$$

Moreover, under (1.9) and (1.10) the closed-loop system achieves the closed-loop poles given from the zeros of $Q C^{p}$.

Remark 1. From a theory of Diophantine equations, given in the Appendix, it is straightforward to show that for arbitrary $Q$ of degree $\delta(Q)$ and $m \geqslant 1$, then a unique solution $R, S$ of (1.9) exists and can be determined with $\delta(R)<m+i, \delta(S)<r+j$, for any positive integers $i, j$ satisfying $(i+j)=\max \{0$, $\delta(Q)+l-(r+m)+1\}$. Moreover, any $R, S$ solution of (1.9) allows a solution for $(A, B)$ of (1.10) if common factors of $R, S$ are also factors of $Q$. With $R, S$ coprime, then a unique $A, B$ solution of (1.10) exists and can be determined with $\delta(A)<\delta(R)+1+i_{*}, \delta(B)<\delta(S)+1+j_{*}$ for any positive integers $i_{*}, j_{*}$ satisfying $\left(i_{*}+j_{*}\right)=\max$ $\{0, \delta(Q)-(\delta(R)+\delta(S)+2)+1\}$. Generic solutions give (in the usual case with $\left.i, j, i_{*}, j_{*}=0\right) \delta(A)=$ 
$\delta(R)=m-1, \delta(B)=\delta(S)=r-1$ with $(R, S)$ coprime and $(A, B)$ coprime. The above degree bounds for the regulator $R, S$ and implicit representation $A, B$ are mildly stronger than the bounds given in Casalino et al. (1985) in terms of $\max \{\delta(R), \delta(S)\}$ and $\max \{\delta(A), \delta(B)\}$.

Remark 2. Consider the closed-loop system (1.1), (1.7) with $R, S$ coprime under (1.9) and (1.10) so that the implicit representation of the closed loop system is (1.8) with $w_{k}$ zero mean, white and with $\sigma_{w}^{2}>0$. Consider that $(A, B)$ is coprime with $\delta(A)$, $\delta(B)$ known as in the generic case. Consider also that $Q C^{p}$ is (strictly) minimum phase so that the closed-loop system with poles at the zeros of $Q C^{p}$ is asymptotically stable. Then recursive least squares (RLS) estimates $\hat{A}_{k}, \hat{B}_{k}$ of appropriate dimension as in (1.6) will globally converge to $A, B$ as $k \rightarrow \infty$. [These results can be viewed as a special case of the ELS results (a posteriori case) for the model (1.3) with $C^{p} \equiv 1$ and (1.2) trivially satisfied.]

\section{$R L S$ based adaptive scheme}

The above Theorem 1.1 and remarks motivate here the study of the convergence properties of an RLS based adaptive scheme. The objective is to achieve convergence of the closed-loop system to an implicit representation as in (1.8). Employ RLS estimation of $A, B$ of (1.8) as $\hat{A}_{k}, \hat{B}_{k}$ via (1.6) of appropriate dimension. Solve for each $k$ the following Diophantine equation for some minimum phase $Q$, or more generally some time-varying $\hat{Q}_{k}$ :

$$
\hat{A}_{k} \hat{R}_{k}-\hat{B}_{k} \hat{S}_{k}=\hat{Q}_{k} .
$$

Then implement the adaptive controller

$$
u_{k}=\hat{S}_{k} \hat{\xi}_{k}, \quad \hat{R}_{k} \hat{\xi}_{k}=y_{k} .
$$

For the case when $\hat{Q}_{k}=Q$, a constant minimum phase polynomial, then the adaptive scheme is in essence an adaptive pole assignment scheme such as in Prager and Wellstead (1980). For the case when $\hat{Q}_{k}=q b_{1}^{-1} \hat{B}_{k}$, then $\hat{R}_{k}=\hat{Q}_{k}$ and $\hat{S}_{k}=\left(\hat{A}_{k}-1\right)$, and with $h_{1}$ a priori specified, the adaptive scheme is seen to be the STR scheme of Åström and Wittenmark (1973). Adaptive LQG schemes can also be viewed in this framework.

The theory of Casalino et al. (1985) also states that should $\hat{Q}_{k}, \hat{A}_{k}, \hat{B}_{k}$ converge to some $\bar{Q}, \bar{A}, \bar{B}$, and the residuals be asymptotically white, then the adaptive controller asymptotically assigns closedloop poles to the zeros of $\bar{Q} \bar{C}^{p}$. It remains to study under what conditions convergence takes place.

In the next section the first steps in developing a convergence theory for the RLS based adaptive pole assignment scheme for ARMAX models are taken. Connections are made to known convergence theory for ELS schemes. We show that the noise condition (1.2) generalizes for the RLS based pole assignment scheme as

$K_{k} \triangleq\left[\left(H_{k} C^{p}\right)^{-1}-\frac{1}{2}\right]$ is strictly passive

$$
\begin{aligned}
H_{k}= & \left(A \tilde{R}_{k}-B \widetilde{S}_{k}-\tilde{Q}_{k}\right)\left(A^{p} R_{k}-B^{p} \tilde{S}_{k}-C^{p} \widetilde{Q}_{k}\right)^{-1} \\
= & \left(\widetilde{B}_{k} \hat{S}_{k}-\tilde{A}_{k} \hat{R}_{k}\right)\left[\left(\hat{A}_{k} \hat{R}_{k}-\hat{B}_{k} \hat{S}_{k}\right) C^{p}\right. \\
& \left.-\left(A^{p} \hat{R}_{k}-B^{p} \hat{S}_{k}\right)\right]
\end{aligned}
$$

where $\tilde{Q}_{k}=Q-\hat{Q}_{k}, \quad \tilde{R}_{k}=R-\hat{R}_{k}, \quad \tilde{S}_{k}=S-\tilde{S}_{k}$. Notice that for the STR of Moore (1985) when $R=Q=q b_{1}^{-1} B, B=B^{p}, S=A-1=\left(A^{p}-C^{p}\right)$ and $\hat{R}_{k}=\hat{Q}_{k}=q b_{1}^{-1} \hat{B}_{k}, \hat{S}_{k}=\hat{A}_{k}-1$, then $\tilde{Q}_{k}=\tilde{R}_{k}$ and from (1.13b), $H_{k}=1$. The passivity condition (1.13a) now simplifies as the strict positive real condition (1.2). However, in contrast to the condition (1.2) which is dependent only on the noise model, the condition (1.13) depends on both the plant and (time-varying) controller parameters. It is therefore sample function dependent. Other interpretations of this condition are given in Section 3.

\section{THE STRICT PASSIVITY CONDITION FOR CONVERGENCE}

Lemma 2.1. The model (1.1) under the controller (1.12) has the representation

$$
\begin{gathered}
A y_{k}=B u_{k}+C_{k} w_{k} \\
C_{k}=\left(A \hat{R}_{k}-B \hat{S}_{k}\right)\left(A^{p} \hat{R}_{k}-B^{p} \hat{S}_{k}\right)^{-1} C^{p} .
\end{gathered}
$$

Moreover, when $\hat{R}_{k}=R, \hat{S}_{k}=S, \hat{Q}_{k}=Q$ and (1.19) is satisfied then $C_{k}=1$.

Proof. From (2.1), $C_{k} w_{k}=\left(A \tilde{R}_{k}-B \hat{S}_{k}\right) \hat{R}_{k}^{-1} y_{k}$. Also from (1.12), $\hat{R}_{k}^{-1} y_{k}=\left(A^{p} \hat{R}_{k}-B^{p} \hat{S}_{k}\right)^{-1} C^{p} w_{k}$. These together give (2.1), (2.2). The second property follows from Theorem 1.1.

Remark. With $Q_{k}, \hat{R}_{k}, \hat{S}_{k}, \hat{A}_{k}, \hat{B}_{k}$ estimates of $Q, R$, $S, A, B$ satisfying (1.9), then an estimate of $C_{k}$, denoted $\hat{C}_{k}$, is $\hat{C}_{k}=C_{k}(R, S)=1$, and a corresponding a posteriori estimate of $w_{k}$ is

$$
\begin{aligned}
\hat{w}_{k / k} & \triangleq y_{k}-\hat{y}_{k / k}, \quad\left(\hat{y}_{k / k}=\psi_{k}^{\prime} \hat{\theta}_{k}\right) \\
& =\left(\widetilde{B}_{k} u_{k}-\tilde{A}_{k} y_{k}\right)+\left(C_{k}-1\right) w_{k}+w_{k} \\
& =\hat{A}_{k} y_{k}-\hat{B}_{k} u_{k}
\end{aligned}
$$

where $\widetilde{B}_{k}=B-\hat{B}_{k}, \widetilde{A}_{k}=A-\hat{A}_{k}$. Likewise an $a$ priori estimate is

$$
\begin{aligned}
\hat{w}_{k / k-1}= & y_{k}-\hat{y}_{k / k-1}=\left(\tilde{B}_{k-1} u_{k}-\tilde{A}_{k-1} y_{k}\right) \\
& +\left(C_{k}-1\right) w_{k}+w_{k}=\hat{A}_{k-1} y_{k}-\hat{B}_{k-1} u_{k} .
\end{aligned}
$$




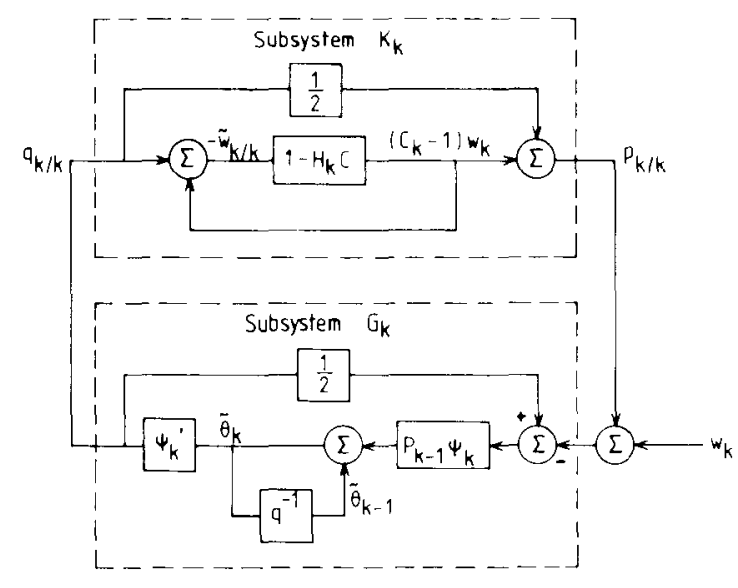

FIG. 1. The error equations $\sim$ a posteriori form.

Standard manipulations from (1.6) give

$$
\begin{aligned}
& \hat{w}_{k / k}=\left(1-\psi_{k}^{\prime} P_{k} \psi_{k}\right) \hat{w}_{k / k-1}, \\
& \left(1+\psi_{k}^{\prime} P_{k-1} \psi_{k}\right) \hat{w}_{k / k}=\hat{w}_{k / k-1} \\
& \tilde{\theta}_{k}=\tilde{\theta}_{k-1}-P_{k-1} \psi_{k} \hat{w}_{k / k} .
\end{aligned}
$$

Lemma 2.2. The parameter estimation error equations (2.5), (2.3) can be organized as two linear subsystems back to back driven by white noise as in Fig. 1. The subsystem with input $q_{k / k}=\psi_{k} \bar{\theta}_{k}$ and output $p_{k / k}=\frac{1}{2} q_{k \mid k}-\left(C_{k}-1\right) w_{k}$ is denoted $K_{k}$ and is defined in (1.13). The subsystem with input $\left(p_{k \mid k}+w_{k}\right)$ and output $q_{k / k}$ is denoted $G_{k}$. Moreover, both subsystems are linear, but time varying.

Proof. Applying (2.1) and (1.2)

$$
\begin{aligned}
\left(C_{k}-1\right) w_{k}= & A y_{k}-B u_{k}-w_{k} \\
= & {\left[A \hat{R}_{k}-B S_{k}-\left(C^{p}\right)^{-1}\left(A^{p} \hat{R}_{k}\right.\right.} \\
& \left.\left.-B^{p} \hat{S}_{k}\right)\right] \xi_{k} \\
= & \left(C^{p}\right)^{-1}\left[C^{p} \hat{Q}_{k}-\left(A^{p} \hat{R}_{k}-B^{p} S_{k}\right) \xi_{k}\right. \\
& \left.+\left(\tilde{A}_{k} \hat{R}_{k}-\tilde{B}_{k} \hat{S}_{k}\right)\right] \xi_{k} \\
= & \left(C^{p}\right)^{-1}\left[H_{k}^{-1}-C^{p}\right]\left(\tilde{B}_{k} \hat{S}_{k}-\tilde{A}_{k} \hat{R}_{k}\right) \xi_{k} \\
= & {\left[\left(H_{k} C^{p}\right)^{-1}\right] q_{k / k} . }
\end{aligned}
$$

Also from (2.3)

$$
\begin{aligned}
\tilde{w}_{k / k} & =w_{k}-\hat{w}_{k / k} \\
& =-\left[q_{k / k}+\left(C_{k}-1\right) w_{k}\right] .
\end{aligned}
$$

Together those yield

$$
\left(C_{k}-1\right) w_{k}=\left(1-H_{k} C^{p}\right)\left(-\tilde{w}_{k / k}\right) .
$$

The relationships of Fig. 1 are now immediate.
Remark 1. The formulation of the estimation error equations as in Fig. 1 now makes possible the application of known ELS convergence theory where in the ELS schemes noise estimates are $a$ posteriori estimates $\hat{w}_{k / k}$ (Kumar and Moore, 1982; Moore, 1983). Some aspects of this theory are recalled in the next few remarks.

Remark 2. An important insight into the behaviour of the error equations of Fig. 1 comes from the result (Kumar and Moore, 1982; Moore, 1983 and their references), that the subsystem $G_{k}$ is passive for arbitrary $\psi_{k}$. Since a passive system in feedback with a strictly passive system driven by $L_{2}$ inputs leads to input-output $L_{2}$ stability, then it makes sense in the context here to assume in a convergence theory that $K_{k}$ is strictly passive as in (1.13). Thus for $w_{k}$ in $L_{2}$, and (1.13) holding,

$$
\sum_{1}^{\infty}\left(p_{k ; k}^{2}+q_{k ; k}^{2}\right)<x, \quad \sum_{1}^{x} \tilde{w}_{k: k}^{2}<\infty .
$$

Also, it can be shown that $\partial_{k}^{\prime} P_{k}{ }^{1} \delta_{k}$ converges. Of course (1.13) is not a necessary condition for input-output stability. Although the subsystems $G_{\mathrm{k}}, K_{\mathrm{k}}$ are time varying, in general, it is helpful to think in terms of "loop gains" at certain frequencies, and "phase shifts" in $G_{k}, K_{k}$ in frequency bands. The assumption that $w_{k}$ is white means that $\psi_{k}$ is colored and with most of its frequency content in the "frequency band" of the closed-loops system. Outside this band, the "loop gain" in Fig. 1 will be "small", so that "phase shifts" of more than 90 could be tolerated in the subsystem $K_{k}$.

Remark 3. A more general stochastic analysis relies on a martingale convergence theorem as in Kumar and Moore (1982) and Moore (1983,1985). To exploit this theorem a modification to the least squares algorithm is required which weights the most recent data less in the presence of instability as detected by ill-conditioning of $P_{k}$. Another key theorem tells us that for linear, reachable (possibly unstable) systems, excitation of inputs (here $u_{k}$ ) translates to excitation of states (here $\psi_{k}$ ) (Moore, 1983). See also Moore and Green (1985) and Moore (1987). With $u_{k}$ persistently exciting, in that its variance is bounded below by a positive value, then

$$
\begin{aligned}
& \lim _{k \rightarrow x} \lambda_{\min } \frac{1}{k} \sum_{1}^{k} \psi_{i} \psi_{i}^{\prime}>0 \\
& \text { (or } \lim _{k \rightarrow \jmath} \inf k P_{k}<x \text { ) a.s. }
\end{aligned}
$$

and

$$
\liminf _{k \rightarrow x} k\left\|\tilde{\theta}_{k}\right\|^{2}<x .
$$




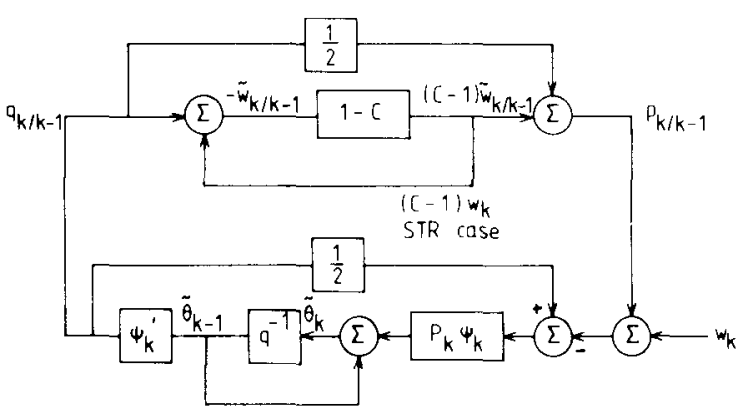

FIG. 2. The error equations (STR case) $\sim$ a priori form.

Remark 4. For the case of the STR of Aström and Wittenmark (1973), where as noted in Section 1, $H_{k}=1$ and (1.13) simplifies as (1.2), the formulation of the error equations in Fig. 1 is novel. The main contribution of this formulation is that it makes a connection with ELS theory based on a posteriori noise estimates $\hat{w}_{k / k}$ rather than on a priori estimates $\hat{w}_{k / k-1}$. The former theory is known to give stronger convergence results, although condition (1.2) is required for both theories. Figure 2 shows the $a$ priori ELS error equations which also apply to the STR. This can be contrasted with the a posteriori form of Fig. 1. The subtle differences are in fact quite significant when it comes to achieving convergence rates and setting rules for the weighting selection scheme. (Of course, since asymptotically the control is a linear combination of the elements in $\psi_{k}$, for $\hat{A}_{k}, \hat{B}_{k}$ to be consistent, one of them must be $a$ priori known and its value prescribed in the least squares algorithm. Typically $b_{1}$ is set $b_{1}=1$ as a scaling.)

Remark 5. In the next section the focus is the strict passivity condition (1.13) which is sample function dependent, in contrast to (1.2) which is only signal model dependent.

\section{INTERPRETATION OF CONVERGENCE CONDITION}

The strict passivity of the subsystem $K_{k}$ of Fig. 1 in the convergence condition (1.13) has an interesting interpretation when $\widetilde{Q}_{k}=0$ and either $\tilde{S}_{k}=0$ or $\tilde{R}_{k}=0$ for all $k$, for then

$$
\begin{aligned}
& {\left[K_{k} \mid \tilde{Q}_{k}=0, \widetilde{S}_{k}=0\right]=\left[A\left(A^{p}\right)^{-1} C^{p}\right]^{-1}-\frac{1}{2}} \\
& {\left[K_{k} \mid \widetilde{Q}_{k}=0, \tilde{R}_{k}=0\right]=\left[B\left(B^{p}\right)^{-1} C^{p}\right]^{-1}-\frac{1}{2} .}
\end{aligned}
$$

These conditions are now dependent only on the signal model.

For adaptive pole assignment $\widetilde{Q}_{k}=0$. Also it is possible to contrive an adaptive scheme such that either $\tilde{S}_{k}=0$ or $\tilde{R}_{k}=0$ for all $k$ so as to test what happens when either of (3.1), (3.2) fail. Consider the case when $\hat{Q}_{k}=Q$ and $\hat{S}_{k}=S$ in (1.11). Then the
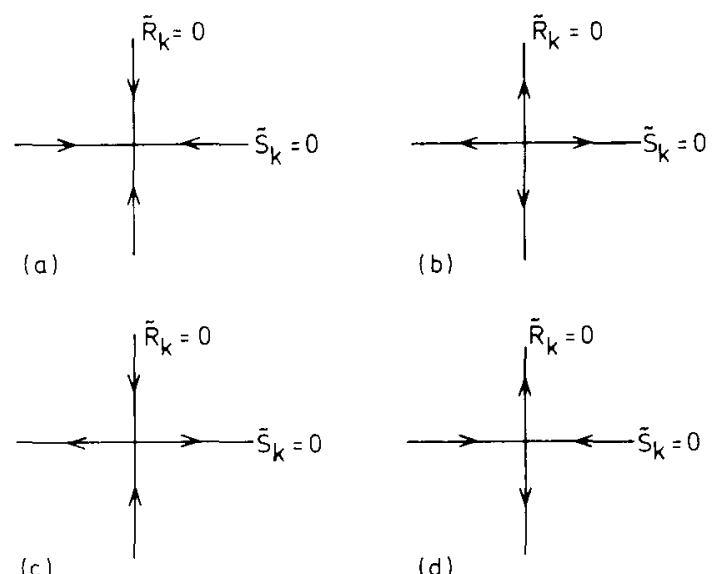

(d)

FiG. 3. The convergence condition interpretation.

adaptive pole assignment controller is

$$
S \hat{R}_{k}^{-1}=S\left(\hat{B}_{k} S+Q\right)^{-1} \hat{A}_{k} .
$$

For this controller, the convergence condition is (3.1). Likewise when $\hat{Q}_{k}=Q$ and $\hat{R}_{k}=S$ in (1.11) then the adaptive controller is

$$
\hat{S}_{k} R^{-1}=\hat{B}_{k}^{-1}\left(\hat{A}_{k} R-Q\right) R^{-1}
$$

and the convergence condition is (3.2).

Simulation studies using the adaptive controller (3.3) applied to the model (1.1), not reported here in detail, show that when (3.1) is satisfied convergence occurs, and when (3.1) is violated there is most often "immediate" instability-even in the white noise case when $C^{p}=1$. This contrasts our simulation studies for adaptive minimum variance controllers for (1.1) when failure of (1.2) does not cause instability, at least in the short term, but merely "drift" in the estimates of parameters.

The diagrams in Fig. 3 are perhaps instructive for more general adaptive schemes based on RLS, at least in interpretation of (1.13). Figure (3a) shows the case when both (3.1), (3.2) are satisfied, Fig. (3b) when both fail, and Figs (3c, d) when one is satisfied and the other fails. Notice that (3.1) fails when the plant is unstable and (3.2) fails when the plant is nonminimum phase. These diagrams do not give any indication of what happens in the entire $\widetilde{R}_{k}$, $\tilde{S}_{k}$ space, and indeed our simulations of adaptive pole assignment schemes do not indicate that entrapment on $\widetilde{R}_{k}=0$, or $\widetilde{S}_{k}=0$ occurs. If $C^{p}$ violates (1.2), then our simulations show similar behaviour to STR schemes when (1.2) is violated.

\section{CONCLUSION}

The theory of the paper has identified a sample function strict passivity convergence condition associated with least squares based adaptor control schemes for ARMAX models in the presence of 
colored noise.

The strict passivity condition, when violated in specially contrived situations, leads to closed-loop instability. In more usual situations, simulations do not suggest that the algorithms become unstable or behave in such a way that the strict passivity condition is violated. Of course instability can occur when the pole assignment controller is itself nonrobust. There is clearly the need for further research on this subject.

Perhaps the most significant result emerging from the new theory pertains to the special case of the STR of Aström and Wittenmark (1973) (adaptive minimum variance control based on least squares identification). The lemmas of this paper now allow a global convergence theory to be expounded for the STR (or rather a mildly modified version) using known theory based on that for extended least squares schemes based on a posteriori noise estimates rather than a priori noise estimates as in earlier studies. The consequent gain is to achieve guaranteed convergence rates of $O(1 / \sqrt{ } k)$ associated with parameter estimate convergence.

\section{REFERENCES}

Anderson, B. D. O. and R. R. Bitmead (1977). Stability of matrix polynomials. Int. J. Control, 26, 235-247.

Åström, K. J. and B. Wittenmark (1973). On self-tuning regulation. Automatica, 9, 185-199.

Casalino, G., F. Davoli, R. Miniciardi and G. Zappa (1985). Adaptive finite-horizon implicit LQ controllers. Proc. IF AC Ident. Syst. Para. Est., York, U.K., July 1985, pp. 1311-1315.

Kumar, R. and J. B. Moore (1982). Convergence of adaptive minimum variance algorithms via weighting coefficient selection. IEEE Trans. Aut. Control, AC-27, 46-153.

Moore, J. B. (1982). Sidestepping the positive real condition for stochastic adaptive schemes. Ric. Aut., 8, 501-523.

Moore, J. B. (1983). Persistance of excitation in extended least square. IEEE Trans. Aut. Control, AC-28, 60-68.

Moore, J. B. (1985). Stochastic adaptive control via consistent parameter estimation. Proc. IFAC Ident. Conf., York, July 1985, pp. 611-616.

Moore, J. B. (1987). A universality advantage of stochastic excitation signals for adaptive control. Syst. Control Lett., to appear.

Moore, J. B. and R. R. Bitmead (1984). On the self-tuning regulations and a priori ELS convergence. Proc. Conf. Decis. Control, FL. Lauderdale, Florida, Dec. 1984

Moore, J. B. and M. Green (1985). Persistence of excitation in linear systems. Proc. America Control Conf., June 1985.
Prager, D. L. and P. E. Wellstead (1980). Multivariable poleassignment self tuning regulators. Proc. IEE, 128D, 9-18.

Ryall, T., J, B. Moore and Lige Xia (1985). Central tendency adaptive control. Proc. IEE Control Conf., Cambridge, July 1985, pp. 116-121. See also, Proc. IEEE CDC Conf., Vol. 1. pp. 100-105, Dec. 1986.

\section{APPENDIX}

On Diophantine equations

The following result builds on those in Anderson and Bitmead (1977), where $R_{s p}$ denotes the class of rational strictly proper transfer functions.

Theorem A.1. Let $A(s), B(s), C_{1}(s), C_{2}(s)$ be prescribed polynomial matrices with $A, B$ coprime and $A^{-1} C_{1}, C_{2} B^{-1} \in R_{s p}$. There exist unique polynomial matrices $X(s) . Y(s)$ satisfying $A X+$ $Y B=C_{1} C_{2}$ with $X B^{-1}, A^{-1} Y \in R_{s}$

Proof. Without loss of generality take $A, B$ as row and column proper, respectively. With $A^{-1} C_{1}, C_{2} B^{-1}$ strictly proper there exist readily determined state-space realizations $A^{-1}(s) C_{1}(s)=$ $H_{1}^{\prime}\left(s I-F_{1}\right)^{-1} G_{1}$, and $C_{2}(s) B^{-1}(s)=H_{2}^{\prime}\left(s I-F_{2}\right)^{-1} G_{2}$. These are such that any strictly proper transfer functions $A^{-1}(s) L(s)$ and $M(s) B^{-1}(s)$ have the form $H_{1}^{\prime}\left(s I-F_{1}\right)^{-1} K_{1}$ and $K_{2}^{\prime}\left(s I-F_{2}\right)^{-1} G_{2}$. Such realizations can always be found. Let $P$ solve $P F_{2}-F_{1} P=-G_{1} H_{2}^{\prime}$. Since $A, B$ are coprime $F_{1}$ and $F_{2}$ have no common eigenvalues so $P$ exists and is readily determined. Now

$$
\begin{aligned}
A^{-1}(s) C(s) B^{-1}(s)=H_{1}\left(s I-F_{1}\right)^{-1} G_{1} H_{2}^{\prime}\left(s I-F_{2}\right)^{-1} G_{2} \\
=H_{1}^{\prime}\left(s I-F_{1}\right)^{-1}\left[P\left(s I-F_{2}\right)\right. \\
\left.\quad-\left(s I-F_{1}\right) P\right]\left(s I-F_{2}\right)^{-1} G_{2} \\
=H_{1}^{\prime}\left(s I-F_{1}\right)^{-1} P G_{2} \\
\quad-H_{1}^{\prime} P\left(s I-F_{2}\right)^{-1} G_{2} \\
=A^{-1}(s) Y(s)+X(s) B^{-1}(s)
\end{aligned}
$$

for some $X, Y$ with $A^{-1} X, Y B^{-1} \in R_{s p}$

Remark 1. Should $A^{-1} C_{1}$ and $C_{2} B^{-1}$ not be strictly proper, then a re-organisation such as $\bar{A} \bar{X}+\bar{Y} \bar{B}=C_{1} C_{2}$ can be performed so that $\bar{A}^{-1} C_{1}, C_{2} \bar{B}^{-1}$ are strictly proper. For example, take $\bar{A}=s^{j} A$ and $X=s^{j} \bar{X}$ and $\bar{B}=s^{i} B, Y=s^{i} \bar{Y}$ for suitably large integers $i, j \geqslant 0$. In the scalar case, it suffices that $A^{-1} C_{1} C_{2} \bar{B}^{-1}$ be proper, so that $(i+j)=\max \left\{0, \delta\left(C_{1} C_{2}\right)-\right.$ $[\delta(A)+\delta(B)]+1\}$

Remark 2. In applying the above results to (1.9), these equations should be first rewritten as, noting $S B^{p}=B^{p} S \equiv$ scalar,

$$
\begin{gathered}
A^{p}(R-1)-S B^{p}=\left(Q C^{p}-A^{p}\right) \\
{[z(A-1)]\left(z^{-1} R\right)-\left(z^{-1} S\right)(z B)=(Q-R)}
\end{gathered}
$$

and then $z^{-1}$ identified with $s$ in the Theorem A.1, so that in the first equation there is the identification $A \sim A^{p}, B \sim B^{p}$, $X \sim(R-1), Y=-S$ and in the second $A \sim z(A-1), B \sim z B$, $X \sim z^{-1} R, Y=-z^{-1} S$. The results of Remark 2 following Theorem 2.1 are then immediate. 